\title{
No one can see me cry: understanding mental health issues for Black and minority ethnic staff in higher education
}

\author{
Jason Arday ${ }^{1}$ \\ Accepted: 28 September 2020 / Published online: 5 January 2021 \\ (c) The Author(s) 2020
}

\begin{abstract}
Black and minority ethnic (BME) communities continue to experience differential outcomes within the United Kingdom (UK) mental health system, despite increased attention on the area. The trauma of racism for BME academic and professional staff within higher education remains problematic against a backdrop of cultural and organisational institutional racism. Within higher education (HE), BME staff consistently face barriers in terms of accessing contextually appropriate mental health interventions that recognise the sophisticated nature of insidious racism in all its overt and covert manifestations. This paper attempts to address the issues facing ethnic minority staff within the Academy with regard to accessing mental health services at university. Importantly, this paper explores the impact of racial discrimination on BME faculty within the sector and the impact upon mental health, in addition to considering the paucity of psychological interventions available in dealing with discriminatory episodes and the need for universities to diversify healthcare professional cohorts. This study utilises the narratives of $40 \mathrm{BME}$ academic and professional university staff to examine the impact of negotiating racial inequality and discrimination at university and the impact upon mental health. Other aspects examined consider the impact of belonging, isolation and marginalisation on mental health and how this consequently affects BME university staff. Conclusions and recommendations provided advocate greater diversification of mental health support systems for BME staff within universities. Conclusions drawn will also consider how existing systems can function to dismantle racial inequality and improve mental health invention for ethnic minority service users.
\end{abstract}

Keywords Black and minority ethnic $(\mathrm{BME}) \cdot$ Ethnic minorities $\cdot$ Service user $\cdot$ Healthcare provider $\cdot$ Racism $\cdot$ Diversification $\cdot$ Inequality

Jason Arday

Jason.a.arday@durham.ac.uk

1 Department of Sociology Higher Education and Social Inequalities, Durham University, 32 Old Elvet, Durham DH1 3HN, UK 


\section{Introduction}

The increasing pressure placed on academics within the higher education sector has led to growing concerns regarding the mental well-being of staff within universities. While there has been much needed widespread attention given towards the issue, there has perhaps been an omission regarding those whose experiences of mental illness are exacerbated by encounters of racism within the Academy. The widespread attention given towards this epidemic has become general in focus rather than contextual in recognising the varying ways in which experiencing racism on a continual daily basis can compromise mental faculties. The continual and residual trauma faced by Black and minority ethnic (BME) ${ }^{1}$ academics requires a particular set of psychological interventions that are situated in helping to unpack the violence of those racialised experiences.

The use of talking and behavioural therapies has been influential in steering individuals away from treatments and interventions that rely solely on medication. Research (Arday, 2018; Grey et al., 2013; Wallace et al., 2016) suggests that BME academics and professional staff continue to experience differential mental health support and psychological interventions in helping to successfully navigate mental illness, particularly in relation to the trauma experienced when facing and navigating racism (Burnett and Peel, 2001; Cooper et al., 2013; Vernon, 2011). There remains a lack of understanding concerning the nuances of discrimination and racism that invariably affect mental well-being, specifically that of ethnic minorities who often are subjected to discriminatory and stereotypical judgements posited by healthcare professionals upon presentation of psychological symptoms or altered mental state.

Within higher education (HE), the issue of mental health and wellness continues to gather momentum, resulting in universities now being required to prioritise this issue as a pastoral imperative, in response to the recent, steadily increasing rate of staff and student suicides (Arday, 2018, 2019). Attempts to recognise the varying contextual wellbeing needs of an ever-broadening university populous have meant a reconceptualisation and adjustment in mental healthcare resource and provision throughout the sector. The residual trauma encountered by BME staff that experience racism and the connection to destabilised mental wellness continues to gain traction. The correlation between continuous encounters of racism within the workplace and the effect on physical and mental wellbeing for BME staff is hardly surprising, particularly given the paucity of psychological interventions available in dealing with this type of trauma (Memon et al., 2016; Myrie and Gannon, 2013).

The physical and mental well-being of BME staff within universities remains an afterthought, which sadly becomes intensified at the height of crisis, often exacerbating feelings of anxiousness, isolation and marginalisation (Palmer and Ward, 2007). The cumulative

\footnotetext{
1 Commentators suggest the use of precise descriptions regarding the ethnic background when describing research findings (Bradby, 2003; McKenzie and Crowcroft, 1996). For the purposes of this paper, the term black and minority ethnic and the abbreviation BME will be used to refer to people who are from ethnic backgrounds other than white British (including Black African, African Caribbean, Asian, Latin-American, and other minority ethnic communities) with more precise descriptions used where appropriate. There is a recognition, however, that the term BME is not universally accepted. Although, this is the term commonly used within the British vernacular. It is important to acknowledge that the term BME, despite its widespread use, has severe limitations and usually follows non-specific quantifiers such as 'most' or 'some' (Glover and Evison, 2009). Typically, there has been an accepted use of the term BME, which has been illustrated in research and Government papers. Given the purpose of this paper, this term is applied purely as a descriptive term having been the preferred term for most of the participants throughout this study.
} 
effect of facing racialised episodes requires further examination as we begin to illuminate discriminatory patterns regarding mental healthcare experiences for ethnic minorities. For many BME staff within higher education, they will often have to navigate the institutionally racist and inequitable terrain of academia. This habitually coincides with a normative and causal integration of insidious and subtle racism, in the form of racial micro-aggressions (a cumulative and covert form of racism involving conscious and intentional actions or slurs) and hyper-surveillance which among many other discriminatory tropes renders ethnic minority individuals as less capable (Rollock, 2012). This context becomes further exacerbated particularly when considering the lofty, egalitarian ideals often espoused by universities (Arday, 2020; Shilliam, 2015). These ideals often become contradicted as universities continue to be complicit, perhaps more than other institutions within British society in maintaining and sustaining racial inequality (Arday, 2019).

The importance of staff diversification extends beyond equity reasons; this also has implications for the often under-represented and actively minoritised BME student population (Arday and Mirza, 2018). Essentially, beyond the moral argument, there is a need for the Academy to be more reflective of a diverse, multi-cultural society (Alexander and Arday, 2015). Universities often espouse and engage in the common belief that diversity in their student bodies, faculties, and staff is important for them to fulfil their primary mission of providing high-quality education (Armstrong, 2008). For students of colour who often become marginalised through exclusionary cultures, the diversification of staff plays a pivotal role in fostering a sense of belonging and enriching educational experiences (Myrie and Gannon, 2013).

Importantly, diversity facilitates a collective challenging of stereotyped preconceptions and encourages critical thinking, in addition to facilitating individuals to be able to communicate and engage effectively with people of varied backgrounds. The need for more diverse university spaces coincides with the one of the main functions of the Academy, preparing individuals to become good citizens in an increasingly complex, pluralistic society (Mirza, 2017). To this end, continuous evaluations of diversification and pastoral interventions are essential within universities, in an attempt to address the paucity and retention of BME academic and professional staff within the sector more generally (Arday, 2019).

This paper examines the impact of negotiating racial inequality and discrimination at universities and the impact upon BME staff mental health. Aspects examined consider the impact of discriminatory cultures which cultivate aspects of victimisation, isolation and marginalisation, resulting in adverse effects on ethnic minority mental health. There is a need to centre the experiences of BME staff within the Academy more generally as, historically, this voice has been omitted from public discourse and debates concerning inequitable spaces. Unpacking this melanoma is paramount in beginning to unearth some of the psychological effects of systemic and sustained racism in the workplace.

The centring of BME experiences regarding mental health is paramount in attempting to examine access to mental health services and comparative engagement. For ethnic minorities, these factors play a significant part in sustaining altered mental states, often exacerbating and compounding the racial oppression experienced (Glover and Evison, 2009; Palmer and Ward, 2007). The victimisation experienced can often be a catalyst towards feelings of isolation and marginalisation, which can be a precursor to altered psychological state (Vernon, 2011). The oppression faced resides within the wider context of enduring institutional racism sustained through hostile and violent cultures. Importantly, this paper will argue that, against a backdrop of continuous racial inequality and discrimination, universities must continuously develop and diversify their pastoral services to deal with more modernised and sophisticated forms of racism that are compromising and altering the 
mental state of BME staff within the higher education sector. The lasting effects, besides the psychological, also impact pathways to career progression, retention and contract security. In considering this destabilising terrain, it is unsurprising that ethnic minorities are at an increased risk of mental health issues (Grey et al. 2013; Williams, 2005; Sewell, 2012; Wilkinson and Pickett, 2010). Understanding the components that impact BME psychological well-being is a central feature in this study, in attempting to discern and illuminate the lasting impact of insidious and systemic racism within the Academy.

Lastly, this paper concludes by providing suggestions and recommendations advocating a need to diversify and modernise mental health services in response to ever-increasing, multi-diverse university communities. Specifically, for ethnic minorities, psychological interventions require a contextual subtlety and understanding from healthcare professionals which allows for BME staff to make sense of their racialised episodes as a remedial and cathartic process. Conclusions drawn will consider how organisational structures and cultures within universities can function to dismantle racism, and alleviate the mental torque placed on BME staff within the Academy. Concluding thoughts will proffer that ethnic minorities encounter sophisticated, cyclical forms of discrimination and institutional racism throughout varying societal structures, which leaves ethnic minorities more susceptible to altered mental states.

\section{Situating the context: the normalisation of racism in the Academy}

The institutional norms of the Academy continue to play a central role with regards to the sustained and racially violent episodes encountered by BME staff. Such cultures are complicit in facilitating racial harassment and psychological abuse. Often, research has indicated (Arday and Mirza, 2018; Miller, 2016) that senior leaders within universities remain consciously or unconsciously complicit in maintaining exclusionary cultures which marginalise and victimise ethnic minorities within the sector. The prevailing discriminatory cultures result in the continuous questioning of professional capabilities regarding BME staff. The Academy has become more so in recent times a cauldron for knowledge production and dissemination, resulting in a more mentally and physically demanding environment and inhabiting biases most notably against minorities and women (Arday, 2020). This is consequential for BME academics in particular who already make up a significant percentage of workers on precarious contracts within the UK higher education sector (Advance HE 2018). Such contexts have significant implications for this particular study due to the fluidity of structural racism that pervades in universities meaning such discriminatory issues continually reside on the periphery and that very little attention is given towards the cumulative impact of racism and the psychological distress encountered by BME staff.

While the premise and focus for this study is BME staff, it is important to acknowledge and recognise that other minorities also continuously face intersectional discrimination within the Academy most notably individuals with disabilities, women and individuals from the LGBQT community. There is an important need to examine the lived discriminatory experiences of these minority groups. Importantly, there continues to be a much needed emerging body of seminal research on these groups. However, contrastingly, there does still remain a dearth of research on the lived racialised experiences of Black people 
particularly within the Academy. This study attempts to centre these experiences through the lens of mental health in education.

\section{Understanding mental health among BME staff within higher education}

The plight of BME mental health within higher education and wider society points to a significant failing of institutional services to successfully support and accommodate ethnic minority patients. Within health-related research, people from ethnic minorities continue to be under-represented, with their experiences absent from the discourse surrounding this context. As a direct consequence, there continues to be an absence of literature which examines the differential nuances of mental health effects on BME communities particularly within professional environments (MIND, 2013). The absence of extensive networks for ethnic minority groups is a pertinent factor in the participation of such mental health research (Davies et al., 2009; Shavers et al., 2001). Attempting to glean narratives and experiences of mental illness within the Academy are problematic due to the cycle of marginalisation and isolation that many BME staff encounter within the workplace environment with self-preservation strategies oscillating from self-regulation to conformity, to survival (Picower, 2009). For many university staff, the intensive gaze of the Academy and the stigma of mental illness often means that individuals are reluctant to disclose any issues regarding mental health (Wilkinson and Pickett, 2010). The backdrop to this particular reoccurring narrative resides within building trust in pastoral interventions provided by universities which do not pre-judge, racially ascribe or label ethnic minorities before cognitive behavioural processes have been utilised (Vernon, 2011). It is important to note that access to university health services (occupational health) will normally be made through either a confidential self-referral or a confidential line manager referral in a duty of care capacity for 'vulnerable' staff. Within universities, most will have separate psychological support and healthcare provision for both students and staff (Arday, 2018).

The perniciousness of racism and its ability to cause continual mental lacerations for BME staff in multiple ways means that there will inevitably be a colouration between the impact of racial discrimination on mental health and physical health. The connectedness between the two factors is a direct result of the continual impact of racism on first the mental and then the physical state. The human and economic cost is best illustrated in the findings of the Racism Ruins Lives report undertaken by Stephen Ashe, Madga Borkowska and James Nazroo (2019). In their report, which surveyed 5000 BME employees across the private, public and voluntary sectors, more than a quarter of the respondents (28\%) who reported experiencing workplace racism stated that they had to take a period of sick leave. Furthermore, a significant proportion of both men and women said that racial discrimination had caused them to leave a job (Ashe et al., 2019).

\section{The generational exclusion of the BME voice in the mental health research}

There has been a lack of trust in research and the medical system more generally by BME communities that have encountered contrasting healthcare experiences or outcomes (Bhui et al., 2004; Sewell, 2012). Shavers et al. (2001) found that often BME individuals are less 
willing to participate in medical research in comparison to their White counterparts often citing misrepresentation and racial ascription as critical reasons. Other practical issues also infiltrate the participation of BME communities within mental health research. Scepticism from BME communities can impact this context significantly, particularly for BME university staff carrying the burden of professional, cultural and communal expectations, while attempting to survive the inequitable terrain of the Academy (Alvarez et al., 2006; Yancey et al., 2006). Other commentaries (Lamb et al., 2012; Rugkåsa and Canvin, 2011) have espoused reasons for the exclusion of BME participants from mental health surveys and interventions. Williams (2005) suggests that some research institutions have established recruitment networks that do not include BME groups. Others (Grey et al., 2013; Memon et al., 2016; Myrie and Gannon, 2013; Williams, 2005) frequently report that additional barriers include language needs and the lack of recognition that healthcare services require modernising to facilitate and accommodate the diverse needs of an ever-increasing multicultural society. The structural inequality that pervades with regards to access of healthcare services becomes an instrument for the continuation of marginalising BME communities who are in need of potential psychological or mental health intervention (Vernon, 2011).

Unpacking this dialogue when situated within a higher education context is challenging, particularly when we consider the ways in which higher education sorts to exclude ethnic minorities (Andrews, 2016; Arday and Mirza, 2018; Arday, 2020). For BME staff within the Academy, the centrality of Whiteness is continuously facilitated through aspects of hyper-surveillance, racial micro-aggressions and a paucity of opportunities to progress professionally (Arday, 2019; Andrews, 2016; Law, 2017; Picower, 2009; Pilkington, 2013). Navigating this inequitable terrain for BME staff can become mentally exhausting and taxing, subsequently impacting on aspects of professional performance within an uncompromising inequitable landscape (Rollock, 2016). Such cumulative experiences regarding the effects of the racial-micro-aggressions and psychological stress responses will also inevitably result in racial battle fatigue (Smith et al., 2007). This term was originally used in reference to the experiences of African American men in America. Its remit has been expanded within racial discrimination vernacular to describe the negative and racially charged experiences of BME people within and beyond the United States (US). Smith et al. (2007) defines racial battle fatigue (RBF) as the cumulative result of a natural race-related stress response to distressing mental and emotional conditions. Further, he states that these conditions emerge from constantly facing racially dismissive, demeaning, insensitive and/or hostile racial environments and individuals. The psychological warfare encountered from of the racial micro-aggression will often result in the erosion of resilience culminating in what Smith describes through the vehicle of RBF as a public health and mental health illness. RBF causes BME individuals to experience various forms of mental, emotional, and physical strain which can lead to psychophysiological symptoms. The cumulative consequences of such symbolic violent acts have led to calls for universities to be cognizant of the implications on the mental wellness of BME staff. Franklin (2019) argues for the need for universities to destigmatise mental health resources and provide counsellors to students (in this case staff) that focus the impact of the racism, racial micro-aggressions and RBF.

Furthermore, this becomes problematic because attitudes towards mental illness within BME communities can be restrictive with discourses often situated in developing greater resilience or establishing a greater connection with faith (religion) as the only reliable intervention in arresting mental health decline (Alvarez et al., 2006; Keating and Robinson, 2004). Keating and Robinson (2004) highlight a societal tendency to trivialise mental illness as mental frailty rather than acknowledging this as a legitimate illness that destabilises individuals' mental state for short or prolonged periods of time. For 
BME individuals' the cultural stigmata associated with mental illness and the history of trivialisation regarding this issue present several difficulties in safely being able to disclose some of the crippling psychological symptoms associated with this illness (Arday, 2018; Bhui et al., 2004; Memon et al., 2016). Aspects of loneliness, marginalisation and isolation can become accelerated as ethnic minorities attempt to deal with this issue on their own ultimately plunging them into further difficulties without the correct coping mechanisms (Keating, 2007). Within a higher education context, ever-expanding university populations mean that pastoral services are continually in high demand, with many healthcare departments severely stretched and under-staffed, resulting in an inevitable reactive rather than pro-active approach towards addressing mental health (Memon et al., 2016). A significant consequence of the sector's growth and expansion has been universities often slow and reactive response towards mental health, with interests firmly fixated on consumer and financial growth (Bhui and Sashidharan, 2003; Fernando, 2003). The reactive aspect within this context recognises the need to attract students and staff as part of the everexpanding university, but fails to recognise the importance of providing support to capably support that expanding and diverse university community.

In some cases within a UK higher education context, the lack of investment in these essential pastoral services weakens universities' abilities to respond to the varying and contextually complex needs of service users (Grey et al., 2013). Illuminating the ethnic minority voice is particularly important in considering how we disrupt dominant monopolies which often omit discourses concerning the BME mental health experience, and more importantly how this pervades within society and the workplace (MIND, 2013; Grey et al., 2013; Rugkåsa and Canvin, 2011). Gleaning this narrative provides a catalyst for reframing and re-imagining discussions regarding mental health amongst ethnic minorities. Wendler et al., (2006) detected very small differences in willingness to take part between ethnic groups in their review of published research that reported consent rates (in mainly quantitative surveys) by race or ethnicity. They concluded that rather than focusing on changing the attitudes of minority groups, it is necessary to engage in a wider debate that aims to recognise the contextual nuances of how particular ethnic groups encounter mental illness, highlighting that this should be the central aim for proliferating health-related research. However, a reconceptualisation of this phenomenon relies on shifting the paradigm for inclusion, which is dependent on a lexicon that prioritises and encourages Black and ethnic minority engagement in disclosing experiences of mental illness (MIND, 2013; Sainsbury Centre for Mental Health, 2002). Fernando (2003) recognises this as an imperative endeavour by asserting that healthcare research must adopt a more inclusive disposition, and in doing so provide more accessible platforms for ethnic minorities to disclose and present symptoms of mental illness. Similarly, it is important to acknowledge that cultural contexts significantly impact upon the presentation and disclosure of destabilised mental health. Establishing modes of trust, inclusion and belonging within the BME community becomes a pivotal component for healthcare practitioners and researchers keen to better support, understand and enable ethnic minorities negotiating this context (Ahmed, 2012).

The progress made regarding greater calls for better representation and diversification within the Academy in terms of wider representation within student services becomes integral particularly in terms of building synergy and familiarity culturally among ethnic minority populations within universities (Arday, 2019; Rollock, 2016; Shilliam, 2015). The well-being of BME academic and professional staff within universities is integral in terms of establishing a sense of belonging, which facilitates integration rather than marginalisation within spaces where often ethnic minorities reside in few numbers (Arday and Mirza, 2018). Lent (2004) situates wellbeing as an essential but often neglected area within psychological health when considering the 
initiation of ethnic minority individuals within universities. Furthermore, Fernando (2010) states that within university spaces a wide variety of factors make it difficult to distinguish the crucial elements of well-being, particularly with regards to negotiating discriminatory cultures which continually place Black and minority ethnic groups at mental and physical risk. Understanding and safeguarding this well-being remains the responsibility of universities, particularly in the case of BME staff who continue to encounter differential experiences regarding professional support and career progression (Alexander, 2018; Law, 2017).

Addressing these factors for universities is imperative because in the UK, research points towards higher rates of mental health problems for BME communities compared with the national average (Cross and Bloomer, 2010; MIND, 2012). The dominant discourse which precedes this context has often situated BME communities with mental health problems to be more likely than others to receive a diagnosis of severe mental illness, to experience involuntary treatment, and more commonly entering the mental health system via the criminal justice system (Keating, 2007; McKenzie et al., 2001; MIND, 2013). The dialogue of severe mental health diagnosis historically inflicted upon ethnic minorities through stereotypical and often racial discrimination has at times resulted in healthcare professionals providing inaccurate and negative summative psychological assessments (Cooper et al., 2013; Memon et al., 2016). Significantly, this has paved the way for a generational distrust of healthcare professionals for many individuals from BME communities, who have sometimes refrained from disclosing any potential mental illnesses for fear of further stigmatisation or racial disadvantage (Keating and Robinson, 2004). Dismantling this lexicon is central to disrupting some of the racially ascribed views held concerning ethnic minority experiences with mental illness (Alvarez et al., 2006; Davies et al., 2009). Fernando (2010) states that there needs to be a wider recognition which acknowledges that when discussing issues concerning mental health with BME communities this particular context has historically been underpinned with managing environments that are toxic and emotionally violent, continuously underpinned by overt and covert occurrences of racism (Department of Health, 2010; McKenzie and Crowcroft, 1996). The emotional toil that accompanies these experiences invariably affects relationships and interactions with professional colleagues within the workplace (Lamb et al., 2012; Wilkinson and Pickett, 2010). This becomes further consequential for ethnic minority individuals that feel isolated in an inequitable space that reinforces racialised traumas encountered (Ahmed, 2012).

\section{Methodology}

For the purpose of this study, fourteen UK-based universities were engaged with, ranging from Russell Group ${ }^{2}$ to Post-92 institutions. ${ }^{3}$ BME academic and professional staff $(n=40)$ aged between 26 and 58 were recruited from a range of university faculties and professional services. Forty semi-structured, open-ended questionnaires were completed in

\footnotetext{
2 'The Russell Group is a self-selected association of 24 public research universities in the United Kingdom. The group is headquartered in London and was established in 1994 to represent its members' interests principally to Government and Parliament. Representing the UK's leading universities, the Russell Group has historically been committed to maintaining its status as research intensive in addition to having unrivaled links with business and the public sector (The Russell Group 2018).

3 New University, synonymous with Post-1992 University or modern university, is a former polytechnic or central institution in the UK that was given university status through the Further and Higher Education Act 1992 or an institution that has been granted university status since 1992 without receiving a royal charter (Armstrong 2008).
} 
addition to two unstructured focus group interviews and forty 60 -min semi-structured individual interviews involving all forty participants to explore lived experiences of negotiating mental illness as BME staff within higher education. The recruitment of participants was facilitated through access to extensive BME academic networks such as the Black British Studies Network and academic and professional communities with a focus on supporting ethnic minority staff within higher education. Further participants were enlisted through a purposeful sampling process which involved recommendations from several ethnic minority colleagues. Geographical considerations and availability were central to the recruitment of participants during this particular phase of the study. Social media platforms were also utilised to enlist BME academic and professional staff. Additionally, convenience sampling was utilised to diversify the pool of participants and responses to ensure that the sample was as representative as possible regarding the broad ethnic minority demographic within the sector to be considered (Cohen et al., 2011; Lamb et al., 2012). The nature of this study was situated within a critical discourse analysis as a way to examine how meanings are represented within particular narratives concerning BME staff mental health in higher education. This research adopts a critical race theory framework to understand both the lived experiences and structural dimensions of institutional racism within the Academy and the psychological impact on mental state. Such conceptual instruments allow for critical insight into the ways in which racism insidiously pervades throughout the sector (Arday, 2019).

The embryonic phase of the study involved each participant being given an anonymous self-administrated questionnaire to complete which was deposited into a ballot box. The questionnaire included information on the following: gender, age (in age groups), academic or professional role, ethnicity (according to the Office of National Statistics classification), marital status and duration of time working within higher education. The study was primarily based on excerpts from the two focus group discussions (each lasting $3 \mathrm{~h}$ in duration) which included 40 participants from the following ethnicities: Asian/Asian British $(n=8)$, Black/Black British $(n=14)$, Mixed-Heritage $(n=13)$ and Latin-American $(n=5)$. All 40 participants were informed about the potentially sensitive and emotional nature of the research. Participants were reminded at this point that in adhering to our duty of care towards them, they were able to withdraw from the research at any stage. This would become particularly pertinent for participants if the study became a trigger towards destabilising, compromising or altering mental state (Memon et al., 2016). In safely and responsibly navigating some of these factors, the professional expertise of mental healthcare professionals was enlisted to deal with the potential level of trauma to be encountered. The study comprised of 24 females and 16 males. The overwhelming majority of participants were the only Black or ethnic minority within their university (Table 1).

The focus groups were conducted in March 2018 within the UK. The objectives of the study were explained to the participants, and informed consent was obtained. Discussions were facilitated by the researcher who had experience in cross-cultural working dynamics and qualitative methods. Mental healthcare professionals were enlisted with experiences in various psychological interventions ranging from behavioural to talking therapies. All focus group sessions and interviews were audio-recorded and transcribed verbatim. In addition to the recorded discussions, written notes were also taken and flipcharts were utilised for participants to engage in mapping and documenting patterns of thought (Davies et al., 2009; Jones and Berry, 1986). Mental healthcare professionals were on hand to facilitate the focus group and any potential traumatic episodes that may have required professional intervention. This phase of the study facilitated a reflexive process which ensured participants' views were clearly documented. Each participant was encouraged to discuss 
Table 1 Participant demographics

\begin{tabular}{llll}
\hline Ethnicity & $\begin{array}{l}\text { Participant } \\
\text { numbers }\end{array}$ & $\begin{array}{l}\text { Academic } \\
\text { staff }\end{array}$ & $\begin{array}{l}\text { Profes- } \\
\text { sional } \\
\text { staff }\end{array}$ \\
\hline Black/Black British & 14 & 8 & 6 \\
Mixed-Heritage & 13 & 6 & 6 \\
Asian/Asian British & 8 & 5 & 4 \\
Latin-American & 5 & 1 & 2 \\
\hline
\end{tabular}

their experiences of racism, the feelings that accompanied this and self-disclose whether this had impacted on their mental wellbeing and health. The supportive and nurturing environment cultivated candid conversations among participants which enriched the disclosure process.

A topic/discussion guide with questions developed and led by the researcher was used to ascertain various aspects of access to mental health services at university and externally within respective local communities; including type of service(s) used, issues with, and experience of using the service, perceived barriers to accessing the service and how healthcare services can be improved for ethnic minorities within university spaces where often BME staff tend to feel marginalised or isolated. Broad topic guide questions included the following: (1) What are your perspectives on mental health? (2) How do ethnic minorities deal with mental health issues? (3) Do you think mental health and psychological services are made accessible to BME academic and professional staff within universities and wider society more generally? (4) Culturally, how might encounters with mental illness differ for ethnic minority men and women in comparison to White people? (5) How can the current mental health/well-being services provided be improved for BME individuals within universities and society more generally? and (6) When do you feel most vulnerable as a BME member of staff and when does this become exacerbated?

Thematic analysis was implemented to identify key themes that were concurrent and commonly emerged amongst the participants, regarding perceived barriers to accessing contextually appropriate mental health services for ethnic minorities at university encountering racism in the workplace (Braun and Clarke, 2006; Braun and Clarke, 2014). The researcher became familiarised with the scripts and notes and developed an iterative coding scheme using NVivo qualitative data analysis software. This process involved the identification of common words and phrases expressed among the participants which were coded and subsequently grouped into themes and sub-themes to illuminate commonalities in experience. Upon identifying key themes that emerged associated with racialised encounters and presentation of psychological symptoms associated with mental illness. Transcripts were coded according to theme. It is important to disclose that during data review process, new themes and sub-themes emerged and were adapted in an iterative process (Braun and Clarke, 2014; Cohen et al., 2011). Establishing positionality and proximity to the research was essential in an attempt to acknowledge and reduce researcher bias. There was some acknowledgement that as a researcher closely associated with racialised discourse, some organic bias may be inherent, although all protocols were administered to ensure objectivity was maintained and any potential biases were minimised throughout the study (Cohen et al., 2011). In an attempt to minimise bias, other researchers and mental health professionals were enlisted for this phase of the data analysis process. The researchers and professionals independently read the scripts and coded and analysed the data to 
enhance the validity of the emerging themes and claims. Anonymised quotes from the participants were used to illustrate pertinent themes identified within the study.

As the researcher, there was a continual reflective process engaged with throughout the study which involved a continual evaluation of approaches, including the potential limitations of approaches utilised due to researcher bias. It is important to state that as the researcher began to establish a professional working relationship with the participants, there was a marked similarity in racialised experiences, resulting in some of the interview and focus group questions becoming 'leading'. While this did not greatly affect the outcome of responses it is important to acknowledge this in an attempt to minimise and recognise that researcher bias was always likely due to the personal proximity of research topic.

\section{Exploring mental health for Black and ethnic minority (BME) staff within higher education}

The study identified two broad inter-related themes as barriers to accessing mental health services adapted from the work of Memon et al. (2016) and Arday (2018) within universities: (1) personal and environmental factors, and (2) relationship between service user and healthcare provider situated around power and hierarchy. The themes identified were comprised into sub-themes which aimed to illuminate some of the perceived problems for ethnic minorities attempting to access psychological intervention and support.

\section{Mental health support available at universities}

In attempting to unpack the paucity of mental health interventions available to ethnic minorities within university spaces, which is the premise for this particular study, it is important to acknowledge the services that are generally available for academic and professional staff. Generally, universities will be able to provide access and signpost university staff to the following external services through consultations with occupational health: counselling, psychological therapies such as cognitive behavioural therapy, and access to mental health support charities such as SANE, The Samaritans, and MIND (MIND 2013; Wallace et al. 2016).

An important consideration acknowledged throughout the proliferation of these findings recognised the inevitable crossover between university mental health provision and National Health Service (NHS) mental health provision. This in part was due to the similarities encountered regarding discriminatory issues across mental healthcare services regarding the presentation of psychological difficulties (Yancey et al, 2006; Wallace et al., 2016). In attempting to discern encounters with mental health issues, the research instrument implemented was not intended to be invasive but rather glean and centre BME experiences when engaging with healthcare professionals regarding mental health within the university space.

There was an intention initially for participants to disclose more candid accounts regarding personal experiences with mental illness at their discretion. The researcher was advised by mental health professionals that the recital or recollection of mental health episodes could be a potential trigger for phases of trauma resulting in the destabilisation of psychological rehabilitation (Grey et al., 2013; McKenzie et al., 2001). The advice and counsel provided suggested that caution would need to be exercised as psychological distress 
could be further exacerbated without professional intervention and support. It was also acknowledged by the researcher that while they had sufficient knowledge in the area of mental health, they were not a qualified healthcare professional and to this end professional support was enlisted in response to recognising the need for specific expertise in dealing with the disclosure of particular traumatic experiences associated with mental health and psychological well-being. It is also important to note that throughout the iterative phases of the research design and participant engagement, all staff had similar experiences of racialisation. All participants expressed difficulties to varying degrees in gaining sufficient and appropriate access to culturally cognizant and suitable psychological intervention within their institutions.

The professional and academic roles of participants had no determinable impact on their access to institutional pastoral services. The most significant impact derived from the exclusionary and discriminatory contexts explored in relation to the impact of racism on mental health and the subsequent absence of pastoral institutional interventions available in dealing with such issues, was the residual trauma experienced from these racially discriminatory episodes. Other intersectional factors such as age, gender or professional role did not point towards positive 'differential' experiences among participants within this particular study. Furthermore, irrespective of age or job description, there was a comprehensive understanding of mental health and wellness among the individuals that participated in this study.

\section{Personal and environmental factors: results and discussion}

Several personal and environmental factors were recognised as affecting access to mental health services for ethnic minorities within universities. Four sub-themes were identified as potential barriers. Each is described below.

\section{Recognition of mental health problems and fear of stigmatisation}

Inability to recognise symptoms of mental illness and the subsequent rejection of those symptoms by some healthcare professionals were mentioned to be key factors in seeking help from mental healthcare services for ethnic minorities (Grey et al., 2013; Keating, 2007). This was particularly pertinent for individuals who were fearful of further stigmatisation professionally and within their communities. For instance, one person stated that:

'Mental illness is a hard thing to accept, I feel that this is a double inflicted trauma when you are Black, because to be frank this is not accepted in our culture. Particularly, because generations before have dealt with racism and what is expected is a continuous show of strength, anything else, or any other emotion is perceived as weakness. Secondly, this also becomes a stick that White people beat you with professionally' (Female, Black, Professional Staff, 2).

This was compounded by a cultural belief that mental health was sometimes not to be discussed openly with family members or faculty from university as there were potential consequences personally and professionally, which could further exacerbate feelings of isolation and victimisation (Norman and Ryrie, 2009). Consequently, seeking mental health 
support or intervention for psychological problems was reported to be difficult, often leading to a crescendo of symptoms at the presentation and crisis stage:

'I have found myself ignoring these symptoms and letting this build and build. The isolation... I experience as a Black person in the Academy is debilitating, you end up residing in this prism which becomes a downward spiral. Consequently, the violence of these racialised cultures affects your mental wellbeing. In my own person case it resulted in a nervous breakdown and me self-harming to gain some semblance of control back that had been taken away from me' (Male, Mixed-Heritage, Academic, 21).

\section{Social networks}

Social networks were considered to be an anchor for BME academic and professional staff who often resided in isolation within university spaces. These feelings were compounded by feelings of marginalisation and feelings of residing on the periphery of social circles within the professional teams and faculties (Wallace et al., 2016). In presenting some of these feelings and cumulative effect of this on mental wellbeing, individuals expressed that healthcare professionals within universities were difficult to talk to when presenting potential symptoms:

'I have worked in my department for 3 years and there has always been a conscious effort by my White colleagues to ostracise me and keep me on the margins of everything. You end up suffering a kind of double violence when you then go and speak to a healthcare professional at university that thinks you are being hyper-sensitive or paranoid, it is really disconcerting and makes you feel even further isolated when you cannot rely on mental health professionals to assist you in your time of need. You say something... and it's like here we go again... Black person talking about race...' (Male, Black, Academic, 27).

Establishing networks with other ethnic minority colleagues that were encountering similar forms of exclusion and victimisation within professional and academic departments became a source of communal support that was considered to be safe, nurturing and cathartic. Establishing trust with healthcare professionals was considered to be difficult because many participants reported a trivialisation of racism as a definite exacerbation of mental illness (Cooper et al., 2013; Grey et al., 2013). There was a feeling that healthcare professionals were not always knowledgeable about the centrality and perniciousness of 'racism' as a subtle, discriminatory instrument. The collective consensus among participants was that there was a chasm in healthcare professionals' knowledge regarding dealing with patients that were continuously encountering racism on a daily basis:

'If it was not for a weekly group we have at my institution which has become a community and a... hub of sorts for BME staff... I think I might have had a breakdown of sorts. Just being able to verbalise to someone that has experienced the violence of racism is a process of healing. Because of the reaction, I have encountered regarding my anxiety and depression and the impact of racism on this when presenting to healthcare professionals, I have often felt that I could not trust them with my illness with regards to supporting from a non-judgemental and ignorant perspective. In many cases, there they could not even empathise or conceptualise what it is like to 
encounter racism on a daily basis in a very subtle and insidious way' (Asian, Female, Professional Staff, 8).

Importantly, the safety of an environment that facilitated openness and belonging was considered an integral factor towards providing a safe space regarding the dissemination of mental health experiences particularly as an ethnic minority. Specifically, it was felt that:

'Having a space to safely disclose these experiences was vital to my self-preservation and sense of not feeling ashamed. To know there were other Black and ethnic minority staff experiencing similar problems with their mental wellness because of racism was sad but empowering because we could lean on each other and get advice on where to source culturally and contextually appropriate counsel...' (Mixed-Heritage, Male, Academic, 14).

However, a context that resurfaced among participants recognised that such 'safe spaces' could become an echo chamber which reverberates cases of mental illness by reliving racialised experiences rather than providing tools to empower and deal with the issues compromising psychological well-being. Through this perspective the idea of 'safe spaces' can be contributory in reliving racially violent episodes. This perhaps speaks to the toxicity of a wider exclusionary problem for people of colour who remain marginalised within the Academy (Leonardo, 2002, 2016). Such therapeutic spaces generally remain 'safer' for White individuals. Leonardo (2002) indicates that safe spaces within university campuses are often hyper-surveilled by White people with regards to people of colour, leading to an intensification and exacerbation of racialised experiences resulting in a 'toxic space' rather than the intended cathartic space. To this end, there were some thoughts which leant towards the potential for these spaces to be counter-productive:

'I recognise the importance of having these spaces but sometimes it can be an even more depressing situation to hear how other colleagues are dealing with this racism and the effect it is having on their mental health. It becomes a bit of a vicious cycle because ideally you want to develop some coping mechanisms for your trauma. But I guess this is what happens when you do not support Black and ethnic minorities experiencing mental illness, they end up having to mobilise and create their own interventions. Universities and healthcare services are massively complicit in this and are to blame...' (Latin-American, Female, Professional Staff, 11).

It was also felt that more of a focus could be placed on making mental health services more accountable by advocating more alternative therapies that recognise the potency and perniciousness of sustained racism as a catalyst for mental illness. One participant highlighted that:

'Mental health services within universities and society more generally for ethnic minorities are poor, there is a one-glove fits all approach that does not acknowledge the type of psychological trauma inflicted by racism and the types of approaches that are required to make sense of those experiences and move forward, as they can be hugely debilitating and destabilising. This can be further exacerbated when engaging with a healthcare professional that has no understanding of these types of plights...' (Black, Male, Academic, 24). 
Further considerations illuminated fears concerning the exploitation of mental illness from White colleagues in narrative associated with BME colleagues not being as professionally capable or competent (Cross and Bloomer, 2010). This resulted in BME individuals within the study feeling a reluctance to utilise pastoral services as concerns emerged that there was potential for this to create further victimisation, compounding an already destabilising experience:

'I am fully aware that as an ethnic minority I am judged sometimes by my White colleagues from a position of deficiency which renders me not as capable. With my experiences with mental illness, I have had to hide it from colleagues and as someone with bipolar disorder my phrases with depression can leave me feelings pretty low. Consistently fighting that against a feeling of not only judgment for my condition but my race... it's exhausting and I am convinced it makes my disorder worse. I am offered opportunities to talk to healthcare professionals at university but they trivialise your experiences by lecturing you on resilience and telling you we all have problems and with you condition what you may perceive as racism, may not actually be racism... it's most likely a hyper-awareness. That says it all really...' (Female, Black, Professional Staff, 28.)

There was a perception that the perceived continual navigation of racial discrimination in higher education required healthcare professionals to engage with continuing professional development, specifically targeted at understanding the psychological impact of racism on ethnic minorities, particularly within a workplace and societal context:

'Mental health has become more complex and now has several strands that require a continuous upskilling and auditing of skills set. I think a lot of the time... mental health services provided at universities quite simply do not have the skills and cultural competence to empathically engage with experiences of racism and the impact on psychological wellbeing. This understanding for me is essential in providing better mental healthcare outcomes for ethnic minorities engaging with this services...' (Mixed-Heritage, Female, Academic, 35).

A strong tenet that was a particularly prominent reference point for colleagues engaged within this study was the importance of informal social support structures, a context highlighted earlier within the study. For participants, this particular mode of support had been generally perceived to be reliable and an enforced alternative to seeking professional mental health services (Arday, 2018; Memon et al., 2016). A feature of this support was tethered to historical and generational feelings of distrust within ethnic minority communities towards healthcare provision:

'As an ethnic minority there is a distrusting of the mental healthcare provision in the UK as this has been an instrument of the state to sustain the oppression of Black and ethnic minorities within the UK. Within certain communities particularly West Indian and African ... there is a distrust of psychiatrists in particular. There is also sometimes a trivialization of mental illness within these communities which can place sole emphasis on prayer as the only cure. I think this can actually be rather restrictive and demonstrates that within our communities we have a lot of work to do also in terms of understanding mental illness to be a real thing...' (Black, Female, Academic, 26). 


\section{Gender differences}

Within the study, several participants noted the difficulties for BME men with regards to disclosing and presenting mental illness. There was a need to discern some of the subtle differences particularly because of high suicide rates in Britain regarding men under the age of 40. There was a feeling that a significant amount of the rhetoric surrounding this had often been presented within a 'White, Eurocentric' narrative that did little to monitor suicide patterns among Black men experiencing mental illness. The line of inquiry at this juncture of the research did not look to preclude female voices, but rather centre Black male voices from the margins as their experiences with psychological wellness are rarely engaged with due to varying forms of toxic masculinity. There was a collective acknowledgement among both female and male participants that, comparatively, the voices of Black men had been silenced on this issue due to societal binaries and often negative portrayals across several intersections (Cooper et al., 2013; Memon et al., 2016). It was felt that historically due to fear of further stigmatisation, there was a reluctance among BME men to discuss or disclose issues aligned to mental health because of the dominant, hypermasculine discourse often associated with Black men. Considerations proffered by participants suggested the potential for further racial ascriptions to be placed on Black men, resulting in further disadvantage when mapped across dominant discourses in the media which often negatively portray Black men:

'As a Black man that has negotiated mental illness, I was always obsessed with not displaying weaknesses... whatever that means?!... I did not want to give leverage to healthcare professionals to place any more judgements on me than they already were. It bad enough that your colleagues know and are casting aspersions on your ability and then putting that on top of the fact they think you are mentally unstable...' (Black, Male, Academic, 32).

'Men kind of prefer keeping things to ourselves ... I always think that, as a Black man, I am judged differently and I am aware that my skin colour means I am always judged differently and in many cases unfairly...' (Male, Mixed-Heritage, Professional Staff, 7).

Participants also perceived that services were not:

'Aware of some of the issues faced by Black men in British society and the impact of persistent racial victimisation encountered on a daily basis in society and the workplace...' (Male, Asian, Professional Staff, 5).

Dominant discourses concerning hyper-masculinity and the societal stereotypes, particularly of Black men were indicated as a barrier towards presenting symptoms of altered mental state:

'Your needs as a Black male regarding mental health can never be separated from the negative, hyper-masculinity chamber society puts us (Black men) in... how are you supposed to be vulnerable within that toxic context and present symptoms of mental illness when you are framed in that way...? It's a vicious cycle...' (Male, Black, Academic, 17).

An extended tenet of this narrative illuminated a perception that culturally and structurally universities were not equipped to deal with BME experiences of mental illness. Participants deemed this to be an additional contributing factor in attempting to navigate and 
access psychological intervention. Thoughts presented also highlighted the potential effect of a mental health diagnosis upon personal and professional contexts:

'In my culture and at work, if someone heard that someone I had a mental illness. . . the labels I would endure, would not only be shameful for me professionally but also personally and another layer of discriminatory and marginalisation, that I just do not have the energy to deal with...' (Male, Asian, Professional Staff, 10).

\section{Relationship between service user and healthcare provider situated around power and hierarchy}

At this juncture of study, it was pivotal to establish the relationship between service users and healthcare providers by considering aspects of power and hierarchy that were recognised as potential barriers towards accessing to mental health services. Three sub-themes were identified as potential barriers. Each is described below.

\section{Language}

Language was seen as a prominent factor among BME staff with regard to accessing mental health services, particularly where English was their second language. Participants emphasised this to be a barrier particularly where there were instances of BME individuals encountering difficulty conversing or understanding English, especially if this was adopted as a second language. The inability to effectively communicate with healthcare professionals proved to be an obstacle in being able to accurately articulate problems. There was an overwhelming consensus that one way in which universities could address this was to employ multi-lingual healthcare professionals from culturally diverse diasporas to accommodate the multi-ethnicity of an ever-increasing diverse Academy:

'As someone relatively new to the United Kingdom (UK), my understanding of English is improving daily. Despite my improvements, I still struggle to converse and make sense sometimes of what others are saying... sometimes I struggle to articulate clearly and accurately what I would like to say to the healthcare professionals provided at university. I am aware of the potential manipulation of my words and meanings... and so sadly to avoid a wrong diagnosis, I just I prefer not to discuss my psychological issues, as my biggest fear is being heavily medicated, which would mean potentially not being able to work and having my visa revoked...' (Female, Latin-American, Female, Academic, 38).

'I think that with all the provision universities have we should definitely have bilingual healthcare professionals. Universities need to respond to their ever-increasing diverse spaces, because the current system is failing Black and Minorities Ethnic individuals at universities... staff and students...' (Male, Black, Academic, 28).

Consequently, the prevailing narrative recognised the additional stress placed on individuals experiencing mental illness and the anxiety this inevitably causes, which may result in withdrawing from healthcare services altogether:

'My fear is receiving an inappropriate investigation into my psychological state, which would compromise my position at work and place me at further risk of dis- 
crimination, in addition to the racial discrimination I already face on a continual basis. For this reason I have taken the decision to manage my mental illness personally...' (Female, Asian, Academic, 23).

Among the participants, they collectively noted that while there was a necessary and increased need for targeted and focused attention on mental wellness within the sector, there was also a need for psychological services that specifically addressed experiences with racial harassment and discrimination in the workplace. The collective rationale for this leant towards the feeling that as racism becomes more sophisticated new tools are required to navigate and dismantle this plight:

'Universities need to wake up to the idea that mental illness is not experienced through one nuance or vacuum there are several ways people experience this, and when you consider the mental trauma people of colour face through experiencing racism... I think we need psychologically specific and appropriate interventions that allow people to make sense of their discriminatory experiences through empowerment and psychological healing...' (Male, Mixed-Heritage, Professional Staff, 33).

\section{Communication: cultural naivety, insensitivity and discrimination}

Participants were explicit in describing how healthcare providers often struggled to understand BME peoples' experiences. Several participants verbalised their frustration in having to continuously explain and forcibly justify racialised encounters:

'Having to repeatedly communicate and explain these experiences kind of just compounds the trauma... it also means if you know that you are going to experience this then you are less likely to utilise these modes of support which can actually compound the experience and effect mental health more...' (Female, Black, Academic, 25).

Participants felt that this lack of understanding by healthcare professionals was problematic and interfered with effective therapy. Furthermore, they also felt that the poor diversification of healthcare professionals within the profession impacted this phenomenon significantly. One participant suggested that:

'The need to diversify this space is so important because for ethnic minorities demonstrating vulnerability... regarding racism, leaves you in a very precarious position as there is always a feeling that this can be used against as another oppressive tool, because let's face it Black people have had their mental health used against you as another oppressive tool to advance ignorant White supremacist ideologies...' (Male, Black, Academic, 37).

Typically, participants also commented on aspects of White fragility and discomfort often encountered when disclosing occurrences of racism with healthcare professionals (DiAngelo, 2018):

'Having to self-censor for fear of upsetting White healthcare professionals is mental taxing and exhausting, this becomes a big problem because you are supposed to use this forum to be open and honest and instead you find yourself managing the feelings of the healthcare professional that is supposed to be helping you navigate this internal angst...' (Female, South Asian, Professional Staff, 24). 
A continual theme that reverberated amongst the participants' alluded to universities and healthcare professionals being unable to conceptualise or empathise with the systemic impact of insidious and visceral racism (MIND, 2013; Palmer and Ward, 2007). The modernising of services to conceptualise and deal with increasingly more sophisticated forms of racism were considered imperative for BME service users to develop confidence in engaging with these psychological interventions and services.

\section{Power and authority}

Among the participants, power and hierarchy were considered as oppressive instruments for maintaining inequality and inequity at the expense of ethnic minorities, resulting in a feeling of not being able to confide in senior leaders within faculties or departments with regards to disclosing potential mental illness:

'Experiencing patronizing and condescending behaviour from senior leaders when you present them with a problem, is only compounded when you see the healthcare professionals and they enact the same behaviour, it leaves you in limbo in terms of dealing with this situation...' (Male, Asian, Academic, 34).

These concerns were compounded by a feeling that healthcare professionals could potentially enforce a hierarchy that creates further inequity for ethnic minorities:

'I think in this situation knowing how you are already characterised racially as a Black person you are hesitant to be candid for fear of being considered clinically unwell resulting in harsher interventions like heavy medicine dosages... or being detained under some mental health act...' (Female, Mixed-Heritage, Academic, 30).

Imbalances of power and hierarchy were considered by participants to be the most challenging factor with regards to mobilising greater diversification in healthcare services at universities and society:

'Addressing power and hierarchy in situation where ethnic minorities are always disadvantaged in the healthcare system requires almost a remodelling of this structure. Hence... the need for better diversification and representation... I think one way to eliminate this power is to have people of colour in these positions. That is not to suggest that power would not still exist but I just think the dynamic changes slightly because if your disclosing experiences of racism to a clinician and they are White sometimes that can compound the experience and end up being counter-productive...' (Black, Female, Professional Staff, 29).

Furthermore, participants also described a sense of helplessness and passivity with regard to healthcare interactions and treatment:

'In this situation because of the way power plays out you cannot disagree with what is being said, you place yourself in a vulnerable situation by doing that as you become aware you are continuously be psychologically assessed in every interaction... that is where the power imbalance becomes really apparent... and as a Black person you are always aware of stoking the fire...' (Female, Black, Academic, 26).

These factors concerning power and hierarchy became a central tenet during the dialogue shared, with a view proffered that universities needed to provide concentrated and concerted efforts into diversifying and widening the range of healthcare professionals on 
offer with particular expertise in dealing with systemic, racialised violence, in attempting to truly accommodate a diverse range of needs and people (Arday, 2020; Arday, 2019; Rollock, 2016; Wallace et al., 2016). Racism was construed amongst the participants as an 'historical and psychological burden', with characteristics associated with power dynamics such as White privilege (Leonardo, 2016). To this end, it was strongly felt through various repetitions that a way of disrupting this particular discriminatory canon was to diversify the profession through greater representation among healthcare professionals with experiences of dealing with racialised trauma, particularly in workplace settings. Furthermore, academic cultures were considered to be complicit in sustaining discriminatory environments in addition to traditions, practices and policies which consequently impact on aspects of wellness and any potential health and well-being interventions concerning this particular endeavour (Mirza, 2017). Through exclusionary mechanisms, power is maintained through privilege and the centring of whiteness often at the expense of BME staff (Arday, 2019; Rollock, 2012).

In attempting to endorse and engage in pro-active interventions to minimise the debilitating impact of racial discrimination, it is important to acknowledge that while the suggested interventions will invariably have varying contextual limitations, the implementation of the following professional development interventions across the sector could be useful in mitigating or addressing pernicious and racially discriminatory experiences; surveys, explorations into exploring workplace harassment, human rights training for selection and review committees which remain largely White in personnel. Future studies and further explorations into BME staff mental health within the workplace could evaluate the potency and effectiveness of such instruments and whether they are effective in firstly, reducing racially discriminatory cultures, and secondly, supporting the psychological rehabilitation of ethnic minorities as a result of encountering systemic and structural racial violence.

\section{Conclusions and recommendations}

The terrain of higher education is undeniably inequitable across several intersections. Racism within the sector continues to persist at the expense of BME professional and academic staff who encounter this oppression through varying and sophisticated instruments of discrimination (Ahmed, 2012; Leonardo, 2016). The perspectives gleaned throughout this study point towards BME professionals encountering differing experiences of mental health services upon presentation of mental illness within universities and society more generally. The need for targeted psychological interventions to better support ethnic minorities in conceptualising and dealing with their racialised experiences is pivotal, in attempting to stay ahead of more sophisticated and pernicious forms of racism (Sewell, 2012; Suresh and Bhui, 2006). The centrality of more insidious forms of racism has meant that the violence encountered within the workplace has a more sustained and detrimental effect on ethnic minorities (Care Quality Commission, 2011; Law, 2017; Sivandan, 1991). The barriers to appropriate intervention are compounded by healthcare professionals not privy to the subtleties of sustained and systemic racism, which can be central to exacerbating forms anxiety, victimisation, depression and isolation.

While a central feature in this paper has been to illuminate BME experiences with mental healthcare professionals, there is an onus on universities to invest resources in diversifying the composition of clinicians to ensure that the service is reflective of a multi-diverse university community. From a societal perspective, collective endeavour 
is required that focuses on removing barriers encountered at the interface between service users and healthcare providers (Vernon 2011). The removal of these barriers upon presentation of psychological symptoms aligned with altered mental state are central to ethnic minorities being able to identify more productive experiences with effective outcomes regarding mental healthcare services. Additionally, raising awareness of mental health within BME communities and recognising that in enduring and surviving such lived experiences bespoke psychological interventions are essential in attempting to encourage health seeking behaviour during the early presentation stages of altered mental state (Dowrick et al. 2009). Importantly, there has been a much needed focus on university staff more generally regarding mental health; however, there is an urgent need to equally prioritise the mental health of BME staff within higher education and reposition their plight from the margins to the centre in acknowledging the pernicious effect of racism (Arday, 2019; Wallace et al., 2016).

The relentless, daily encounter with racial discrimination is a nuanced and complex experience that requires contextual psychological interventions such as cognitive behavioural therapy, mindfulness-based cognitive therapy or eye movement desensitisation and reprocessing (Lamb et al., 2012; Sewell, 2012). Such interventions must coincide with a more diverse pool of healthcare professionals aware of the impact of racism and attempts must be made where prevalent to not decentre racism as the problem. Silencing and reducing the severity of these racialised experiences has huge implications for BME staff seeking psychological intervention, particularly in recounting such occurrences to colleagues, line managers or mental health professionals; often this can be traumatic and triggering further compounding the mental distress encountered (Lamb et al., 2012; Rugkasa and Canvin, 2011).

Healthcare professionals should be supported to undertake continuing professional development that facilitates an understanding concerning the various types of intersectional discrimination. This training must focus on how ethnic minority groups are affected by racism and the impact on mental state more specifically within universities. Tangible actions can be implemented through the following practical recommendations: raising awareness of mental health issues while reducing stigma, raising awareness of services and access pathways, diversifying healthcare staff within the mental health services, and developing cultural cognizance and sensitivity (Grey et al. 2013). Government interventions and policies tasked with tackling mental health must consider how mental health support systems can diversify. Within a university and societal context, considerations are needed in discerning how workplace structures and cultures function to sustain racism. Examining the inequitable terrain is paramount because BME individuals continue to be more likely to be at risk of mental illness and less likely to receive the appropriate support required (BME Network 2016).

Within a higher education context BME staff experience mental health differently. These experiences are often exacerbated by racially violent and hostile environments within the workplace. The importance of more productive psychological outcomes for ethnic minorities is essential in establishing a mental health system that satisfies and accommodates the plethora of diverse service users (Vernon 2011). Moving forward, systemic changes are required to dismantle the centrality of institutional racism, in creating spaces that allow individuals experiencing mental illness from all types of diverse backgrounds to access the psychological intervention and support they are entitled to receive. 
Funding This research was supported by MIND

Open Access This article is licensed under a Creative Commons Attribution 4.0 International License, which permits use, sharing, adaptation, distribution and reproduction in any medium or format, as long as you give appropriate credit to the original author(s) and the source, provide a link to the Creative Commons licence, and indicate if changes were made. The images or other third party material in this article are included in the article's Creative Commons licence, unless indicated otherwise in a credit line to the material. If material is not included in the article's Creative Commons licence and your intended use is not permitted by statutory regulation or exceeds the permitted use, you will need to obtain permission directly from the copyright holder. To view a copy of this licence, visit http://creativecommons.org/licenses/by/4.0/.

\section{References}

Advance HE. (2018) Equality in higher education, Staff statistical report 2018, available at: https://www. ecu.ac.uk/publications/equality-higher-education-statistical-report-2018/

Ahmed, S. (2012) On being included: Racism and diversity in institutional life. Durham \& London: Duke University Press.

Alexander, C. (2018) Breaking black: The death of ethnic and racial studies in Britain. Ethnic and Racial Studies, 41(6), 1034-1054.

Alexander, C. \& Arday, J. (2015) Aiming Higher Race, Inequality and Diversity in the Academy. London: AHRC: Runnymede Trust, (Runnymede Perspectives). London, Common Creative: Runnymede Trust, (Runnymede Perspectives).

Alvarez, R. A., Vasquez, E., Mayorga, C. C., Feaster, D. J., \& Mitrani, V. B. (2006). Increasing minority research participation through community organization outreach. Western Journal of Nursing Research, 28(5), 541-560. https://doi.org/10.1177/0193945906287215

Andrews, K. (2016) Black Studies University Course Long Overdue. Retrieved from: https://www. theguardian.com/commentisfree/2016/may/20/black-studies-university-course-long-overd

Arday, J. (2018). Understanding mental health: What are the issues for black and ethnic minority students at university? Social Sciences, 7(10), 196.

Arday, J. (2019). Dismantling power and privilege through reflexivity: negotiating normative Whiteness, the Eurocentric curriculum and racial micro-aggressions within the Academy. Whiteness and Education, $3(2), 141-161$.

Arday, J. (2020) Race, education and social mobility: We all need to dream the same dream and want the same thing. Educational Philosophy and Theory, 10, 1-7.

Arday, J., \& Mirza, H. (Eds.). (2018). Dismantling Race in Higher Education: Racism, Whiteness and Decolonising the Academy. London: Palgrave Macmillan.

Ashe, S., Borkowska, M., \& Nazroo, J. (2019). An analysis of the 2016-2017 Trade Union Congress Racism at Work Survey. Centre on Dynamics of Ethnicity: University of Manchester.

Armstrong, C. (2008) What is a University in the UK? Retrieved from: https://web.archive.org/web/20100513000141/ http://www.jobs.ac.uk/careers/articles/1135/what-is-a-university-in-the-uk/

Bhui, K., McKenzie, K., \& Gill, P. (2004). Delivering mental health services for a diverse society. British Medical Journal, 329(7462), 363-364. https://doi.org/10.1136/bmj.329.7462.363

Bhui, K., \& Sashidharan, S. P. (2003). Should there be separate psychiatric services for ethnic minority groups? British Journal of Psychiatry, 182, 10-12.

BME Network (2016) NHS BME network. Retrieved from: https://www.nhsbmenetwork.org.uk

Bradby, H. (2003). Describing ethnicity in health research. Ethnicity and Health, 8(1), 5-13. https://doi. org/10.1080/13557850303555

Braun, V., \& Clarke, V. (2006). Using thematic analysis in psychology. Qualitative Research in Psychology, 3, 77-101.

Braun, V., \& Clarke, V. (2014). What can 'thematic analysis' offer health and wellbeing researchers? International Journal of Qualitative Studies on Health and Wellbeing, 9(2), 121-132.

Burnett, A., \& Peel, M. (2001). Health needs of asylum seekers and refugees. British Medical Journal, 322, 544-557.

Care Quality Commission. (2011) Count Me In 2010 census. Retrieved from https://www.cqc.org.uk/organisationswe-regulate/mental-health-services/count-me-2010-census

Cohen, L., Manion, L., \& Morrison, K. (2011). Research methods in education (7th ed.). Abingdon: Routledge.

Cooper, C., Spiers, N., \& Livingston, G. (2013). Ethnic inequalities in the use of health services for common mental disorders in England. Social Psychiatry and Psychiatric Epidemiology, 48, 685-692. 
Cross, W. M., \& Bloomer, M. J. (2010). Extending boundaries: clinical communication with culturally and linguistically diverse mental health clients and carers. International Journal of Mental Health Nursing, 19(4), 268-277.

Davies, B., Larson, J., Contro, N., Reyes-Hailey, C., Ablin, A. R., Chesla, C. A., \& Cohen, H. (2009). Conducting a qualitative culture study of pediatric palliative care. Qualitative Health Research, 19, 5-16. https://doi.org/10.1177/1049732308327346

Department of Health. (2010). Delivering race equality action plan: A five year review. Retrieved from: https://www.nmhdu.org.uk/silo/files/race-equality-action-plan-a-five-year-review.pdf

DiAngelo, R. (2018). White fragility: Why it's so hard for white people to talk about racism. USA: Beacon Press.

Dowrick, C., Gask, L., \& Edwards, S. (2009). Researching the mental health needs of hard-to-reach groups: managing multiple sources of evidence. BMC Health Serv Res, 9, 226.

Fernando, S. (2003). Cultural diversity, mental health and psychiatry: The struggle against racism. Hove, England: Brunner-Routledge.

Fernando, S. (2010). Mental health, race and culture. London, England: Palgrave Macmillan.

Franklin, J. D. (2019). Coping with racial battle fatigue: differences and similarities for African American and Mexican American college students. Race Ethnicity and Education, 22(5), 589-609. https://doi. org/10.1080/13613324.2019.1579178

Glover, G., \& Evison, F. (2009). Use of new mental health services by ethnic minorities in England. Durham, England: North East Public Health Observatory.

Grey, T., Sewell, H., Shapiro, G., \& Ashraf, F. (2013). Mental health inequalities facing UK minority ethnic populations: Causal factors and solutions. Journal of Psychological Issues in Organizational Culture, 3(1), 146-157.

Jones G. Berry M. (1986) Regional secure units: the emerging picture In: Edwards G (Ed) Current issues in clinical psychology IV London Plenum Press

Keating, F. (2007) African and African Caribbean men and mental health. Better Health Briefing Paper 5. Retrieved from https://www.raceequalityfoundaiton.org.uk/

Keating, F., \& Robertson, D. (2004). Fear, black people and mental illness: A vicious circle. Health and Social Care in the Community, 12(5), 439-447.

Lamb, J., Bower, P., \& Rogers, A. (2012). Access to mental health in primary care: a qualitative meta-synthesis of evidence from the experience of people from 'hard to reach' groups. Health (London), 16, 76-104.

Law, I. (2017). Building the Anti-racist University, action and new agendas. Race Ethnicity and Education, 20(3), 332-343.

Leonardo, Z. (2002). The souls of white folk: Critical pedagogy, whiteness studies, and globalization discourse. Race Ethnicity \& Education, 5(1), 29-50. https://doi.org/10.1080/13613320120117180

Leonardo, Z. (2016). The color of supremacy. In E. Taylor, D. Gillborn, \& G. Ladson-Billings (Eds.), Foundations of critical race theory in education (2nd ed., pp. 265-277). New York: Routledge.

Lent, R. W. (2004). Toward a unifying theoretical and practical perspective on well-being and psychosocial adjustment. Journal of Counselling Psychology, 51(4), 482-463.

McKenzie, K., \& Crowcroft, N. S. (1996) Describing race, ethnicity, and culture in medical research. British Medical Journal, 312 (7038), 1054. Retrieved from https://www.bmj.com

McKenzie, K., Samele, C., van Horn, E., Tatten, T., van Os, J., \& Murray, R. B. (2001) Comparison of the outcome of the treatment of psychosis for people of Caribbean origin living in the UK and British Whites: Report from the UK700 trial. British Journal of Psychiatry, 178 (2): 160-165. Retrieved from: https://bjp.rcpsych.org

Memon, A., Taylor, K., Mohebati, L. M., Sundin, J., Cooper, M., Scanlon, T., \& de Visser, R. (2016). Perceived barriers to accessing mental health services among Black and Minority Ethnic (BME) communities: A qualitative study in Southeast England. British Medical Journal Open, 6(11), e012337.

Miller, P. (2016). 'White sanction', institutional, group and individual interaction in the promotion and progression of black and minority ethnic academics and teachers in England. Power and Education, $8(3), 205-221$.

MIND (2012) Statistics 3: Race, culture and mental health [Fact sheet]. Retrieved from https://www. mind.org.uk/help/people_groups_and_communities/statistics_3_race_culture_and_mental_health

MIND. (2013). Mental health crisis care: commissioning excellence for Black and Minority Ethnic groups (p. 2013). London: MIND.

Mirza, H. S. (2017) 'One in a million: A journey of a post-colonial woman of colour in the white academy', in Deborah Gabriel and Shirley Anne Tate (Eds). Inside the Ivory Tower: Narratives of Women of colour surviving and thriving in academia. London: Trentham UCL Press. 
Myrie, C. V., \& Gannon, K. N. (2013). "Should I really be here?” Exploring the relationship between Black men's conceptions of wellbeing, subject positions and help-seeking behaviour. Diversity and Equality in Health and Care, 10(1), 1-22.

Norman, I., \& Ryrie, I. (2009). The art and science of mental health nursing: Reconciliation of two traditions in the cause of public health. International Journal of Nursing Studies, 46(12), 1537-1540.

Palmer, D., \& Ward, K. (2007). 'Lost': listening to the voices and mental health needs of forced migrants in London. Medicine, Conflict and Survival, 23, 198-212.

Picower, B. (2009). The unexamined whiteness of teaching: How white teachers maintain and enact dominant racial ideologies. Race Ethnicity and Education, 12(2), 197-215.

Pilkington, A. (2013). The interacting dynamics of institutional racism in higher education. Race, Ethnicity and Education, 16(2), 225-245.

Rollock, N. (2016) How much does your university do for racial equality? Retrieved from: https://www. theguardian.com/higher-education-network/2016/jan/19/how-much-does-your-university-do-for-racialequality/

Rugkåsa, J., \& Canvin, K. (2011). Researching mental health in minority ethnic communities: Reflections on recruitment. Qualitative Health Research, 21(1), 132-143.

Sainsbury Centre for Mental Health. (2002) Breaking the circles of fear: A review of the relationship between mental health services and African and Caribbean communities. London, England: Sainsbury Centre for Mental Health. Retrieved from https://www.centreformentalhealth.org.uk/pdfs/breaking_ the_circles_of_fear.pdf

Sewell, $\bar{H}$. (2012). Race and ethnicity in mental health care. In P. Phillips, T. Sandford, \& C. Johnston (Eds.), Working in mental health: Policy and practice in a changing environment (pp. 104-115). Oxon: Routledge.

Shavers, V., Lynch, C., \& Burmeister, L. (2001) Racial differences in factors that influence willingness to participate in medical research studies. Annals of Epidemiology, 12 (4): 248-256. Retrieved from https:// www.annalsofepidemiology.org

Shilliam, R. (2015) Black Academia in Britain. The Disorder of Things, Retrieved from: https://thedisorderofthings. com/2014/07/28/black-academia-in-britain/

Sivandan A. (1991) Black struggles against racism In Curriculum Development Project Steering Group (Eds) Setting the context London England CCETSW.

Smith, W. A., Allen, W. R., \& Danley, L. L. (2007). 'Assume the position ... You fit the description': Psychosocial experiences and racial battle fatigue among African American Male College Students. American Behavioural Scientist, 51(4), 511-578.

Suresh, K., \& Bhui, K. (2006). Ethnic minority patients' access to mental health services. Psychiatry, 5, 413-416.

The Russell Group (2018) What are we About? Retrieved from: https://russellgroup.ac.uk/about/

Vernon, P. (2011) Put race equality in mental health back on the agenda. Retrieved from: https://www.guardian. co.uk/society/joepublic/2011/mar/01/race-equality-mental-health

Wallace, S., Nazroo, J., \& Bécares, L. (2016). Cumulative effect of racial discrimination on the mental health of ethnic minorities in the United Kingdom. American Journal of Public Health, 106(7), 1294-1300.

Wendler, D., Kington, R., Madans, J., Van Wye, G., Christ-Schmidt, H., \& Pratt, L. A. (2006). Are racial and ethnic minorities less willing to participate in health research? Public Library of Science Medicine, 3(2), 19-27. https://doi.org/10.1371/journal.pmed.0030019

Wilkinson, R., \& Pickett, K. (2010). The spirit level: Why equality is better for everyone. London, England: Penguin Books.

Williams, C. C. (2005). Ethical considerations in mental health research with racial and ethnic minority communities. Community Mental Health Journal, 41(5), 509-520. https://doi.org/10.1007/s10597005-6358-y

Yancey, A. K., Ortega, A. N., \& Mumanyika, S. K. (2006). Effective recruitment and retention of minority research participants. Annual Review of Public Health, 27, 1-28. https://doi.org/10.1146/annurev. publhealth.27.021405.102113

Rollock, N. (2012). Unspoken rules of engagement: navigating racial micro-aggresions in the academic terrain. International Journal of Qualitative Studies in Education, 25 (5): 517-532.

Publisher's Note Springer Nature remains neutral with regard to jurisdictional claims in published maps and institutional affiliations. 